\title{
Construction of the Global Solutions to the Perturbed Riemann Problem for the Leroux System
}

\author{
Pengpeng Ji and Chun Shen \\ School of Mathematics and Statistics Science, Ludong University, Yantai, Shandong 264025, China \\ Correspondence should be addressed to Chun Shen; shenchun3641@sina.com
}

Received 25 June 2016; Accepted 18 September 2016

Academic Editor: Ming Mei

Copyright ( 92016 P. Ji and C. Shen. This is an open access article distributed under the Creative Commons Attribution License, which permits unrestricted use, distribution, and reproduction in any medium, provided the original work is properly cited.

\begin{abstract}
The global solutions of the perturbed Riemann problem for the Leroux system are constructed explicitly under the suitable assumptions when the initial data are taken to be three piecewise constant states. The wave interaction problems are widely investigated during the process of constructing global solutions with the help of the geometrical structures of the shock and rarefaction curves in the phase plane. In addition, it is shown that the Riemann solutions are stable with respect to the specific small perturbations of the Riemann initial data.
\end{abstract}

\section{Introduction}

We are concerned with the Leroux system [1] which is represented in the form

$$
\begin{aligned}
u_{t}+\left(u^{2}+\rho\right)_{x} & =0, \\
\rho_{t}+(\rho u)_{x} & =0,
\end{aligned}
$$

in which $u$ and $\rho$ stand for the velocity and density, respectively. It was shown in [2] that system (1) can be derived as a hydrodynamic limit under Eulerian scaling for a twocomponent lattice gas. The Leroux system (1) has been widely used in various fields; for example, it may be utilized to investigate the stochastic dynamics in the stochastic particle system [3] and a deposition growth in the biological chemotaxis-mechanism model [4].

It is easy to see that the Leroux system (1) is strictly hyperbolic in the upper-half plane $\rho \geq 0$ provided that $(u, \rho) \neq(0,0)$ for both the eigenvalues of system (1) are distinct. In addition, the Leroux system (1) is genuinely nonlinear for it is so in both of the characteristic fields. The main feature of system (1) lies in the fact that the $k$-shock curves $(k=1,2)$ coincide with the $k$-rarefaction curves in the $(u, \rho)$ phase plane attributed to the special structure of system (1). Thus, system (1) belongs to the so-called Temple class [5]. In comparison with general systems of hyperbolic conservation laws, the well-posed result for the Temple class can be obtained for a much larger class of initial data $[6$, 7]. For the related works about the Leroux system (1), the entropy solutions were obtained in [8] when the initial data were taken in the form of the sum of Dirac measures and bounded variation functions. The global existence of weak solutions to the Cauchy problem for system (1) was obtained in [9] by constructing four families of Lax-type entropies and entropy fluxes and in [10] by using a new technique from the div-curl lemma in the compensated compactness theorem. In addition, the global bounded entropy solution of system (1) was also achieved in [11] for the bounded measurable initial data by combining the kinetic formulation and the compensated compactness method.

In this paper, we are interested in constructing the global solutions in a fully explicit form to the particular Cauchy problem for system (1) when the initial data are taken to be three piecewise constant states as

$$
(u, \rho)(x, 0)= \begin{cases}\left(u_{-}, \rho_{-}\right), & -\infty<x<0, \\ \left(u_{m}, \rho_{m}\right), & 0<x<x_{0}, \\ \left(u_{+}, \rho_{+}\right), & x_{0}<x<+\infty,\end{cases}
$$

in which $x_{0}>0$ is arbitrarily small. System (1) is of interest for the reason that it is one of the simplest, nonstrictly hyperbolic systems of Temple class. Some useful information 
for Temple class can be achieved by studying the particular Cauchy problem (1) and (2). This type of initial data (2) has been widely used such as in [12-14] to study the wave interaction problem [15-19] for different hyperbolic systems of conservation laws. It is worthwhile to notice that the initial data (2) may be regarded as the specific small perturbations of the corresponding Riemann initial data:

$$
(u, \rho)(x, 0)= \begin{cases}\left(u_{-}, \rho_{-}\right), & -\infty<x<0, \\ \left(u_{+}, \rho_{+}\right), & 0<x<+\infty .\end{cases}
$$

Thus, the particular Cauchy problem (1) and (2) is called as the perturbed Riemann problem (or the double Riemann problems) below.

To deal with the perturbed Riemann problem (1) and (2) it is essential to study various possible interactions of elementary waves for the Leroux system (1). We find that there exist 16 different combinations of Riemann solutions at the initial points $(0,0)$ and $\left(x_{0}, 0\right)$. It is clear that the interaction between the 2-wave starting from $(0,0)$ and the 1-wave starting from $\left(x_{0}, 0\right)$ happens at first which will play a critical role in the construction of solution to the perturbed Riemann problem (1) and (2). It is well known in [20] that only the existence result can be obtained by studying the Goursat problem and the global solution cannot be constructed in a completely explicit form for the perturbed Riemann problem (1) and (2) when the 2-rarefaction wave from $(0,0)$ and the 1rarefaction wave from $\left(x_{0}, 0\right)$ occur. Thus, some assumptions should be taken to avoid the above situation. If the interaction between the 2 -wave from $(0,0)$ and the 1 -wave from $\left(x_{0}, 0\right)$ is completed, then the remaining problem is just to study the interaction between the waves of the same family which is easy to be dealt with for the Temple class. For simplicity, we restrict ourselves to considering the above situation; that is, the solution of the Riemann problem at the origin $(0,0)$ is two shock waves $S_{1}+S_{2}$. In order to meet the above requirements, let us make the following assumption.

Assumption 1. Assume that $u_{m}<u_{-}$together with

$$
\begin{gathered}
\max \left(0, \frac{\left(\sqrt{u_{-}^{2}+4 \rho_{-}}-u_{-}\right)\left(u_{m}-u_{-}\right)}{2}+\rho_{-}\right)<\rho_{m} \\
<\frac{\left(-u_{-}-\sqrt{u_{-}^{2}+4 \rho_{-}}\right)\left(u_{m}-u_{-}\right)}{2}+\rho_{-} ;
\end{gathered}
$$

then the solution of the Riemann problem originating from the origin $(0,0)$ is two shock waves for the perturbed Riemann problem (1) and (2).

In fact, we want to investigate the perturbed Riemann problem (1) and (2) by fixing the Riemann solutions from $(0,0)$ and then changing the Riemann solutions from $\left(x_{0}, 0\right)$. That is to say, for the given left state $\left(u_{-}, \rho_{-}\right)$, we first fix the intermediate state $\left(u_{m}, \rho_{m}\right)$ and then change the right state $\left(u_{+}, \rho_{+}\right)$. With the method described above, we have the following theorem to describe the main result of this paper.
Theorem 2. If the initial data (2) satisfy Assumption 1, then the global solutions of the perturbed Riemann problem (1) and (2) can be constructed in a completely explicit form. Furthermore, the limits of the global solutions to the perturbed Riemann problem (1) and (2) are identical with the corresponding ones of the Riemann problem (1) and (3) when the limit $x_{0} \rightarrow 0$ is taken.

The wave interaction problem for the Temple class has been widely investigated recently, such as for the pressureless gas dynamics equations [21], the isentropic Chaplygin gas dynamics equations $[22,23]$, the Aw-Rascle traffic flow model [14], and various types of chromatography systems [2427]. It is worthwhile to notice that all the above systems are not genuinely nonlinear, in which at least one of the characteristic fields is linearly degenerate. Thus, the wave interaction problem for the above systems has relatively simple structures. Otherwise, both of the characteristic fields are genuinely nonlinear for the Leroux system (1), which is obviously different from the above systems, such that the wave interaction problem is more difficult to be dealt with. To our knowledge, the wave interaction problem for the system of Temple class with two genuinely nonlinear characteristic fields has not been paid attention to before.

We can investigate the wave interaction problem for system (1) in the explicit form by combining the method of characteristics together with the geometrical structures of the rarefaction and shock curves in the $(u, \rho)$ phase plane when the initial data are taken to be three piecewise constant states (2). The global solutions of the perturbed Riemann problem (1) and (2) are constructed completely through obtaining the exact result of each interaction during the process of constructing the solutions under our assumption, which is able to reveal more properties of system (1) than those of the solutions to the Riemann problem (1) and (3). Furthermore, we can see that the solutions of the Riemann problem (1) and (3) are stable with respect to the small perturbations (2) of the Riemann initial data (3) if the limit $x_{0} \rightarrow 0$ is taken.

The paper is organized in the following way. In Section 2, the Riemann solutions of (1) and (3) are restated for convenience. In Section 3, the wave interaction problems are investigated widely for system (1). Under the suitable assumptions, the global solutions of the perturbed Riemann problem (1) and (2) are constructed in explicit forms when the initial data are taken to be three piecewise constant states. In the end, the stability of Riemann solutions is analyzed with respect to the specific small perturbation of the Riemann initial data and the conclusion is drawn in Section 4.

\section{The Riemann Problem for Leroux System (1) and (3)}

In this section, we are dedicated to recalling some main results about the Riemann problem for the Leroux system (1) with the Riemann initial data (3), which has been extensively investigated, for example, in $[1,8]$. We can also refer to such as $[15,28-31]$ for the general knowledge about the Riemann problem for hyperbolic systems of conservation laws. 
By a simple calculation, it is shown that system (1) has two characteristic velocities (eigenvalues):

$$
\begin{aligned}
& \lambda_{1}=\frac{3 u-\sqrt{u^{2}+4 \rho}}{2}, \\
& \lambda_{2}=\frac{3 u+\sqrt{u^{2}+4 \rho}}{2} .
\end{aligned}
$$

Thus, system $(1)$ is strictly hyperbolic in the upper-half $(u, \rho)$ phase plane $(\rho \geq 0)$ provided that $(u, \rho) \neq(0,0)$. The corresponding right eigenvectors for system (1) are

$$
\begin{aligned}
& \overrightarrow{r_{1}}=\left(1, \frac{-u-\sqrt{u^{2}+4 \rho}}{2}\right)^{T}, \\
& \overrightarrow{r_{2}}=\left(1, \frac{\sqrt{u^{2}+4 \rho}-u}{2}\right)^{T} .
\end{aligned}
$$

It is easy to get $\nabla \lambda_{i} \cdot \vec{r}_{i}=2 \neq 0(i=1,2)$, in which $\nabla=(\partial / \partial u, \partial / \partial \rho)$. Thus, both of the characteristic fields are genuinely nonlinear provided that $(u, \rho) \neq(0,0)$, which implies that (1) is a genuinely nonlinear system except for the origin. The waves associated with the two characteristic fields will be either shock waves or rarefaction waves which are determined by the choice of initial data. The Riemann invariants along with the characteristic fields may be selected as

$$
\begin{aligned}
& \omega_{1}=\frac{-u-\sqrt{u^{2}+4 \rho}}{2}, \\
& \omega_{2}=\frac{-u+\sqrt{u^{2}+4 \rho}}{2} .
\end{aligned}
$$

For smooth solutions, by taking the self-similar transformation $\xi=x / t$, system (1) may be rewritten in the form

$$
\left(\begin{array}{cc}
2 u-\xi & 1 \\
\rho & u-\xi
\end{array}\right)\left(\begin{array}{l}
u \\
\rho
\end{array}\right)=\left(\begin{array}{l}
0 \\
0
\end{array}\right) .
$$

For the given left state $\left(u_{-}, \rho_{-}\right)$, the two rarefaction wave curves can be expressed, respectively, by

$$
\begin{aligned}
& R_{1}\left(u_{-}, \rho_{-}\right):\left\{\begin{array}{l}
\xi=\lambda_{1}=\frac{3 u-\sqrt{u^{2}+4 \rho}}{2}, \\
\rho-\rho_{-}=\left(\frac{-u_{-}-\sqrt{u_{-}^{2}+4 \rho_{-}}}{2}\right)\left(u-u_{-}\right),
\end{array}\right. \\
& R_{2}\left(u_{-}, \rho_{-}\right):\left\{\begin{array}{l}
\xi=\lambda_{2}=\frac{3 u+\sqrt{u^{2}+4 \rho}}{2}, \\
\rho-\rho_{-}=\left(\frac{\sqrt{u_{-}^{2}+4 \rho_{-}}-u_{-}}{2}\right)\left(u-u_{-}\right) .
\end{array}\right.
\end{aligned}
$$

It is clear to see that both of the rarefaction wave curves are straight lines in the $(u, \rho)$ phase plane. By direct calculation, one finds that $u_{\xi}=1 / 2>0$ and $\rho_{u}<0$ for the 1-rarefaction wave and $u_{\xi}=1 / 2>0$ and $\rho_{u}>0$ for the 2-rarefaction wave. Thus, the 1-rarefaction wave is made up of the half-branch of $R_{1}\left(u_{-}, \rho_{-}\right)$with $u \geq u_{-}$and $\rho \leq \rho_{-}$, while the 2 -rarefaction wave is made up of the half-branch of $R_{2}\left(u_{-}, \rho_{-}\right)$with $u \geq u_{-}$ and $\rho \geq \rho_{-}$.

On the other hand, for discontinuous solutions, the Rankine-Hugoniot conditions at a discontinuous curve $x=$ $x(t)$ can be expressed as

$$
\begin{aligned}
-\sigma[u]+\left[u^{2}+\rho\right] & =0, \\
-\sigma[\rho]+[\rho u] & =0,
\end{aligned}
$$

where $\sigma=d x / d t$ and $[u]=u_{r}-u_{l}$ with $u_{l}=u(x(t)-0, t)$ and $u_{r}=u(x(t)+0, t)$, and so forth, which implies that

$$
\frac{\rho_{r}-\rho_{l}}{u_{r}-u_{l}}=\frac{-u_{l} \pm \sqrt{u_{l}^{2}+4 \rho_{l}}}{2} .
$$

Then, for the given left state $\left(u_{-}, \rho_{-}\right)$, the two shock wave curves can also be expressed, respectively, by

$$
\begin{aligned}
& S_{1}\left(u_{-}, \rho_{-}\right):\left\{\begin{array}{l}
\frac{x}{t}=\sigma_{1}=u+\frac{u_{-}-\sqrt{u_{-}^{2}+4 \rho_{-}}}{2}, \\
\rho-\rho_{-}=\left(\frac{-u_{-}-\sqrt{u_{-}^{2}+4 \rho_{-}}}{2}\right)\left(u-u_{-}\right),
\end{array}\right. \\
& S_{2}\left(u_{-}, \rho_{-}\right):\left\{\begin{array}{l}
\frac{x}{t}=\sigma_{2}=u+\frac{u_{-}+\sqrt{u_{-}^{2}+4 \rho_{-}}}{2}, \\
\rho-\rho_{-}=\left(\frac{\sqrt{u_{-}^{2}+4 \rho_{-}}-u_{-}}{2}\right)\left(u-u_{-}\right) .
\end{array}\right.
\end{aligned}
$$

It is remarkable that both of the shock wave curves are also straight lines in the $(u, \rho)$ phase plane. One can see that the classical Lax entropy condition implies that $\rho>\rho_{-}$and $u<$ $u_{-}$for the 1-shock wave curve and $\rho<\rho_{-}$and $u<u_{-}$for the 2-shock wave curve.

It is clear that the shock curves coincide with the rarefaction curves in the phase plane. Thus, system (1) belongs to the so-called Temple class [5]. If the first- and second-family wave curves are denoted by $W_{1}\left(u_{-}, \rho_{-}\right)=S_{1}\left(u_{-}, \rho_{-}\right) \cup R_{1}\left(u_{-}, \rho_{-}\right)$ and $W_{2}\left(u_{-}, \rho_{-}\right)=S_{2}\left(u_{-}, \rho_{-}\right) \cup R_{2}\left(u_{-}, \rho_{-}\right)$, respectively, then the lines of $W_{1}\left(u_{-}, \rho_{-}\right)$and $W_{2}\left(u_{-}, \rho_{-}\right)$are just tangent to the parabola $\left\{\Gamma: u^{2}+4 \rho=0\right\}$ in the $(u, \rho)$ phase plane. Furthermore, it can be seen that the slopes of the two tangent lines originating from $\left(u_{-}, \rho_{-}\right)$to the parabola $\left\{\Gamma: u^{2}+4 \rho=0\right\}$ are exactly calculated by the corresponding Riemann invariants in formula (7). Let us draw Figure 1 in the $(u, \rho)$ phase plane to illustrate the situation. For the given left state $\left(u_{-}, \rho_{-}\right)$, in view of the right state $\left(u_{+}, \rho_{+}\right)$in the different regions, the solutions of the Riemann problem (1) and (3) can be summarized as follows: $S_{1}+S_{2}$ when $\left(u_{+}, \rho_{+}\right) \in \mathrm{I}\left(u_{-}, \rho_{-}\right), S_{1}+$ $R_{2}$ when $\left(u_{+}, \rho_{+}\right) \in \operatorname{II}\left(u_{-}, \rho_{-}\right), R_{1}+S_{2}$ when $\left(u_{+}, \rho_{+}\right) \in \operatorname{III}\left(u_{-}\right.$, $\left.\rho_{-}\right)$, and $R_{1}+R_{2}$ when $\left(u_{+}, \rho_{+}\right) \in \operatorname{IV}\left(u_{-}, \rho_{-}\right)$. 


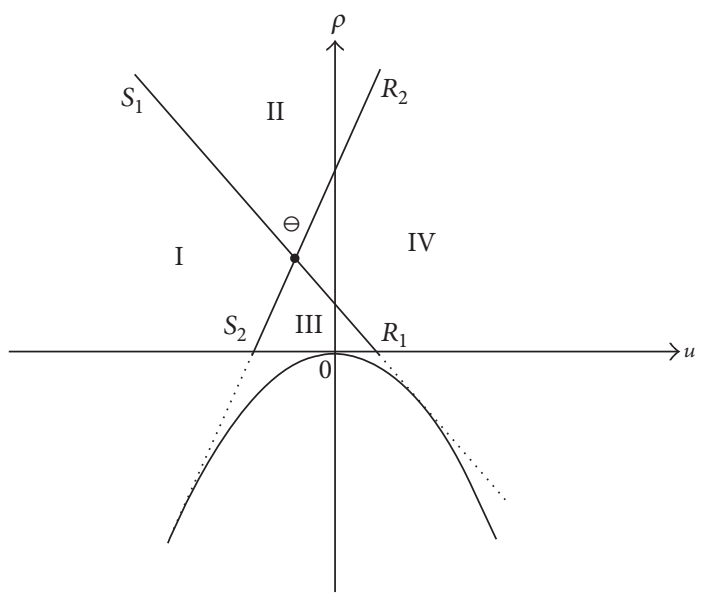

FIGURE 1: For the given left state $\left(u_{-}, \rho_{-}\right)$, the half-upper $(u, \rho)$ phase plane is shown for the Leroux system (1).

For simplicity, let us introduce the notations,

$$
\begin{aligned}
& k_{1}^{-}=\frac{-u_{-}-\sqrt{u_{-}^{2}+4 \rho_{-}}}{2}, \\
& k_{2}^{-}=\frac{-u_{-}+\sqrt{u_{-}^{2}+4 \rho_{-}}}{2},
\end{aligned}
$$

to stand for the slopes of the two tangent lines originating from $(u, \rho)$ to the right-hand and left-hand sides of the parabola $\left\{\Gamma: u^{2}+4 \rho=0\right\}$ in the $(u, \rho)$ phase plane.

For the Riemann problem (1) and (3), the intermediate state $\left(u_{*}, \rho_{*}\right)$ is the one which can be connected with $\left(u_{-}, \rho_{-}\right)$ on the left-hand side by 1-wave and can be connected with $\left(u_{+}, \rho_{+}\right)$on the right-hand side by 2 -wave. No matter what the Riemann initial data (3) are taken, we always have the following relations:

$$
\begin{aligned}
& k_{1}^{-}=\frac{-u_{-}-\sqrt{u_{-}^{2}+4 \rho_{-}}}{2}=\frac{-u_{*}-\sqrt{u_{*}^{2}+4 \rho_{*}}}{2}=k_{1}^{*}, \\
& k_{2}^{*}=\frac{-u_{*}+\sqrt{u_{*}^{2}+4 \rho_{*}}}{2}=\frac{-u_{+}+\sqrt{u_{+}^{2}+4 \rho_{+}}}{2}=k_{2}^{+} .
\end{aligned}
$$

The similar notations have also been adopted here and below and we do not give the detailed explanation any more without confusion. Thus, it can be seen that the intermediate state $\left(u_{*}, \rho_{*}\right)$ can be calculated by

$$
\begin{gathered}
\left(u_{*}, \rho_{*}\right)=\left(\frac{k_{1}^{-} u_{-}-k_{2}^{+} u_{+}+\rho_{+}-\rho_{-}}{k_{1}^{-}-k_{2}^{+}}, \rho_{-}\right. \\
\left.+\frac{k_{1}^{-}\left(k_{2}^{+}\left(u_{-}-u_{+}\right)+\rho_{+}-\rho_{-}\right)}{k_{1}^{-}-k_{2}^{+}}\right),
\end{gathered}
$$

for all kinds of Riemann initial data (3) due to the special form of system (1).

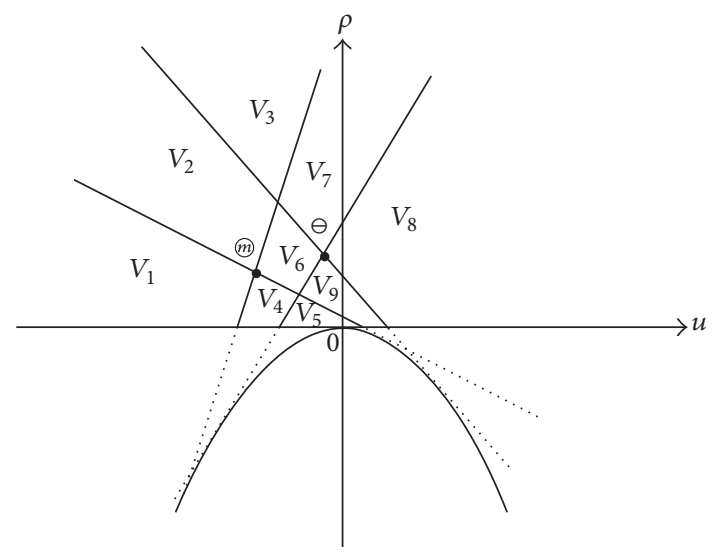

FIgURE 2: If $\left(u_{m}, \rho_{m}\right) \in \mathrm{I}\left(u_{-}, \rho_{-}\right)$, then the nine different regions in the half-upper $(u, \rho)$ phase plane are shown for the perturbed Riemann problem (1) and (2).

\section{Construction of Global Solutions to the Perturbed Riemann Problem (1) and (2)}

In this section, the main purpose is to construct the global solutions of the perturbed Riemann problem (1) and (2) under Assumption 1. In other words, we assume that the Riemann solution starting from the origin $(0,0)$ is always two shock waves. In this situation, we will construct the global solutions of the perturbed Riemann problem (1) and (2) case by case by investigating the wave interaction problems in detail. If we put all the elementary wave curves across the states $\left(u_{-}, \rho_{-}\right)$and $\left(u_{m}, \rho_{m}\right)$ together, then the upper-half $(u, \rho)$ phase plane is divided into nine different regions shown in Figure 2. According to the different Riemann solutions originating from the other initial point $\left(x_{0}, 0\right)$, our discussion may be divided into the following four cases.

Case $1(S+S$ and $S+S)$. We first consider the situation that there are also two shock waves emitting from the other initial point $\left(x_{0}, 0\right)$. Obviously, the occurrence of this case depends on the conditions $u_{+}<u_{m}$ and

$$
\begin{aligned}
& \max \left(0, \rho_{m}+\frac{\left(\sqrt{u_{m}^{2}+4 \rho_{m}}-u_{m}\right)\left(u_{+}-u_{m}\right)}{2}\right)<\rho_{+} \\
& <\rho_{m}+\frac{\left(-u_{m}-\sqrt{u_{m}^{2}+4 \rho_{m}}\right)\left(u_{+}-u_{m}\right)}{2} .
\end{aligned}
$$

For convenience, we use $S_{3}$ and $S_{4}$ to denote the two shock waves, respectively. In this case, the state $\left(u_{+}, \rho_{+}\right)$should locate in the region $V_{1}$ in Figure 2 only. When the time $t$ is small enough, the solution of the perturbed Riemann problem (1) and (2) may be represented succinctly as (see Figure 3)

$$
\begin{aligned}
& \left(u_{-}, \rho_{-}\right)+S_{1}+\left(u_{1}, \rho_{1}\right)+S_{2}+\left(u_{m}, \rho_{m}\right)+S_{3} \\
& \quad+\left(u_{2}, \rho_{2}\right)+S_{4}+\left(u_{+}, \rho_{+}\right) .
\end{aligned}
$$



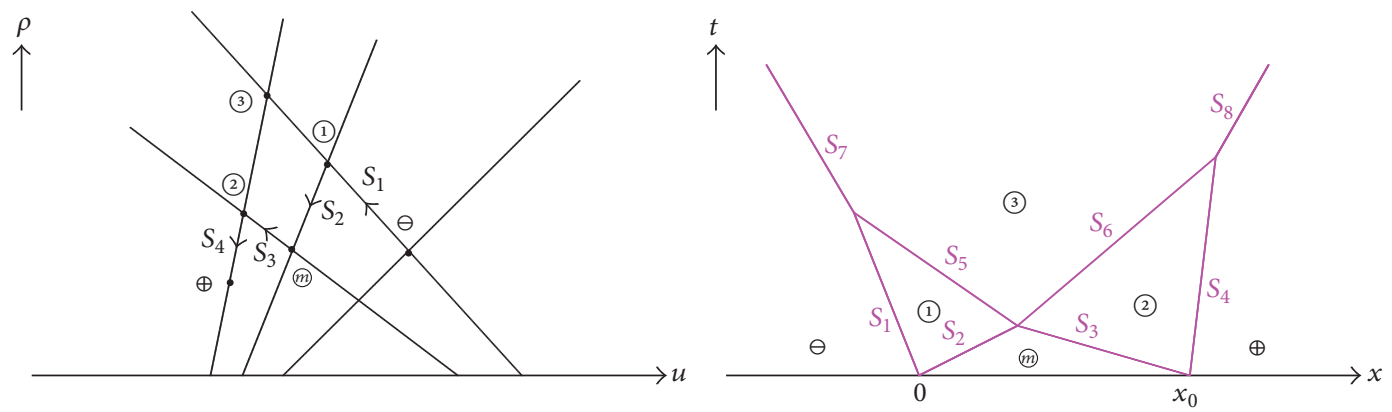

FIgURE 3: The interaction between $S+S$ and $S+S$ is displayed when $\left(u_{+}, \rho_{+}\right) \in V_{1}$.

It follows from (17) that the intermediate states $\left(u_{1}, \rho_{1}\right)$ and $\left(u_{2}, \rho_{2}\right)$ may be given, respectively, by

$$
\begin{gathered}
\left(u_{1}, \rho_{1}\right)=\left(\frac{k_{1}^{-} u_{-}-k_{2}^{m} u_{m}+\rho_{m}-\rho_{-}}{k_{1}^{-}-k_{2}^{m}}, \rho_{-}\right. \\
\left.+\frac{k_{1}^{-}\left(k_{2}^{m}\left(u_{-}-u_{m}\right)+\rho_{m}-\rho_{-}\right)}{k_{1}^{-}-k_{2}^{m}}\right), \\
\left(u_{2}, \rho_{2}\right)=\left(\frac{k_{1}^{m} u_{m}-k_{2}^{+} u_{+}+\rho_{+}-\rho_{m}}{k_{1}^{m}-k_{2}^{+}}, \rho_{m}\right. \\
\left.+\frac{k_{1}^{m}\left(k_{2}^{+}\left(u_{m}-u_{+}\right)+\rho_{+}-\rho_{m}\right)}{k_{1}^{m}-k_{2}^{+}}\right),
\end{gathered}
$$

in which $k_{1}^{-}$and $k_{2}^{+}$are given by (16) and $k_{1}^{m}$ and $k_{2}^{m}$ are calculated, respectively, by

$$
\begin{aligned}
& k_{1}^{m}=\frac{-u_{m}-\sqrt{u_{m}^{2}+4 \rho_{m}}}{2}, \\
& k_{2}^{m}=\frac{-u_{m}+\sqrt{u_{m}^{2}+4 \rho_{m}}}{2} .
\end{aligned}
$$

It can be seen from Figure 3 that the relations $u_{+}<u_{2}<u_{m}<$ $u_{1}<u_{-}, \rho_{1}>\max \left(\rho_{m}, \rho_{-}\right)$, and $\rho_{2}>\max \left(\rho_{m}, \rho_{+}\right)$can be established directly.

Lemma 3. The shock wave $S_{2}$ collides with the shock wave $S_{3}$ in finite time.

Proof. The propagation speeds of $S_{2}$ and $S_{3}$ can be computed, respectively, by

$$
\begin{gathered}
\sigma_{2}=u_{m}+\frac{u_{1}+\sqrt{u_{1}^{2}+4 \rho_{1}}}{2}=u_{m}-k_{1}^{1}, \\
\sigma_{3}=u_{2}+\frac{u_{m}-\sqrt{u_{m}^{2}+4 \rho_{m}}}{2}=u_{2}-k_{2}^{m} .
\end{gathered}
$$

It is obvious to get $u_{m}>u_{2}$ and $k_{1}^{1}<0<k_{2}^{m}$ from Figure 3. Thus, we have

$$
\sigma_{2}-\sigma_{3}=u_{m}-u_{2}-k_{1}^{1}+k_{2}^{m}>0,
$$

which implies that $\sigma_{2}>\sigma_{3}$. That is to say, the shock wave $S_{2}$ collides with $S_{3}$ in finite time. The intersection $\left(x_{1}, t_{1}\right)$ is determined by

$$
\begin{aligned}
x_{1} & =\sigma_{2} t_{1}, \\
x_{1}-x_{0} & =\sigma_{3} t_{1},
\end{aligned}
$$

which yields

$$
\begin{aligned}
& \left(x_{1}, t_{1}\right) \\
& \quad=\left(\frac{\left(u_{m}+u_{1}+k_{2}^{1}\right) x_{0}}{u_{1}-u_{2}+u_{m}+2 k_{2}^{1}}, \frac{x_{0}}{u_{1}-u_{2}+u_{m}+2 k_{2}^{1}}\right) .
\end{aligned}
$$

The collision between the two shock waves $S_{2}$ and $S_{3}$ occurs at the point $\left(x_{1}, t_{1}\right)$, where we again have a local Riemann problem with the initial data $\left(u_{1}, \rho_{1}\right)$ and $\left(u_{2}, \rho_{2}\right)$. In order to solve this problem, we must determine the relative position of $\left(u_{2}, \rho_{2}\right)$ based on $\left(u_{1}, \rho_{1}\right)$ in the $(u, \rho)$ phase plane which can be described below.

Lemma 4. The state $\left(u_{2}, \rho_{2}\right)$ lies in region I with respect to the state $\left(u_{1}, \rho_{1}\right)$. In other words, the solution of the local Riemann problem at the point $\left(x_{1}, t_{1}\right)$ is also two shock waves.

Proof. With $u_{2}<u_{m}<u_{1}$ and $\rho_{m}<\min \left(\rho_{1}, \rho_{2}\right)$ in mind, in order to show that the state $\left(u_{2}, \rho_{2}\right)$ lies in region I with respect to the state $\left(u_{1}, \rho_{1}\right)$ in the $(u, \rho)$ phase plane, it is sufficient to show that the line $S_{1}\left(u_{m}, \rho_{m}\right)$ always lies below the line $S_{1}\left(u_{1}, \rho_{1}\right)$. It can be obtained from (13) that

$$
\begin{aligned}
& S_{1}\left(u_{1}, \rho_{1}\right): \rho-\rho_{1}=\left(\frac{-u_{1}-\sqrt{u_{1}^{2}+4 \rho_{1}}}{2}\right)\left(u-u_{1}\right) \\
& \quad=k_{1}^{1}\left(u-u_{1}\right) \\
& S_{1}\left(u_{m}, \rho_{m}\right): \rho-\rho_{m} \\
& \quad=\left(\frac{-u_{m}-\sqrt{u_{m}^{2}+4 \rho_{m}}}{2}\right)\left(u-u_{m}\right) \\
& =k_{1}^{m}\left(u-u_{m}\right) .
\end{aligned}
$$


Noticing that $u_{1}>u_{m}, \rho_{1}>\rho_{m}$ and $k_{1}^{1}<k_{1}^{m}<0$, one can easily obtain that the inequality

$$
k_{1}^{1}\left(u-u_{1}\right)+\rho_{1}-k_{1}^{m}\left(u-u_{m}\right)-\rho_{m}>0
$$

holds for $u<u_{m}<u_{1}$.

Therefore, it can be concluded from Section 2 that the solution of the Riemann problem with the initial data $\left(u_{1}, \rho_{1}\right)$ and $\left(u_{2}, \rho_{2}\right)$ at the point $\left(x_{1}, t_{1}\right)$ is also two shock waves. Let us use $S_{5}$ and $S_{6}$ to denote them, respectively (see Figure 3). Analogously, the intermediate state $\left(u_{3}, \rho_{3}\right)$ between $S_{5}$ and $S_{6}$ can also be obtained by

$$
\begin{gathered}
\left(u_{3}, \rho_{3}\right)=\left(\frac{k_{1}^{1} u_{1}-k_{2}^{2} u_{2}+\rho_{2}-\rho_{1}}{k_{1}^{1}-k_{2}^{2}}, \rho_{1}\right. \\
\left.+\frac{k_{1}^{1}\left(k_{2}^{2}\left(u_{1}-u_{2}\right)+\rho_{2}-\rho_{1}\right)}{k_{1}^{1}-k_{2}^{2}}\right),
\end{gathered}
$$

in which $\left(u_{1}, \rho_{1}\right)$ and $\left(u_{2}, \rho_{2}\right)$ are given by (20) and (21), respectively. In addition, the relations $k_{1}^{1}=k_{1}^{-}$and $k_{2}^{2}=k_{2}^{+}$ can be established easily. Thus, the conclusion may be drawn.

More precisely, let us first compare the propagation speeds of $S_{3}$ and $S_{5}$ and then compare those of $S_{2}$ and $S_{6}$, respectively. In a word, we use the following lemma to state the results.

Lemma 5. The inequalities $\sigma_{3}<\sigma_{5}$ and $\sigma_{2}>\sigma_{6}$ can be established for the corresponding propagation speeds of shock waves.

Proof. The propagation speeds of $S_{3}$ and $S_{5}$ are calculated, respectively, by (24) and

$$
\sigma_{5}=u_{3}+\frac{u_{1}-\sqrt{u_{1}^{2}+4 \rho_{1}}}{2}=u_{3}-k_{2}^{1} .
$$

Taking into account $u_{2}<u_{3}$ and $k_{2}^{m}=k_{2}^{1}$, we have

$$
\sigma_{3}-\sigma_{5}=u_{2}-u_{3}<0 \text {. }
$$

Thus, the shock wave $S_{3}$ decelerates backward when it passes through $S_{2}$.

On the other hand, the propagation speeds of $S_{2}$ and $S_{6}$ are calculated, respectively, by (23) and

$$
\sigma_{6}=u_{2}+\frac{u_{3}+\sqrt{u_{3}^{2}+4 \rho_{3}}}{2}=u_{2}-k_{1}^{3} .
$$

Noticing that $u_{2}<u_{m}$ and $k_{1}^{1}=k_{1}^{3}$, we also have

$$
\sigma_{2}-\sigma_{6}=u_{m}-u_{2}>0 \text {. }
$$

Thus, the shock wave $S_{2}$ decelerates forward when it passes through $S_{3}$.

Now, we are in the position to consider the coalescence of two shock waves belonging to the same family which is shown below.
Lemma 6. The two shock waves $S_{1}$ and $S_{5}$ belonging to the first family coalesce into a new shock wave of the first family. Similarly, the two shock waves $S_{6}$ and $S_{4}$ belonging to the second family coalesce into a new shock wave of the second family.

Proof. We start with the interaction between $S_{1}$ and $S_{5}$. The propagation speed of $S_{1}$ is

$$
\sigma_{1}=u_{1}+\frac{u_{-}-\sqrt{u_{-}^{2}+4 \rho_{-}}}{2}=u_{1}-k_{2}^{-},
$$

which, together with (31), yields

$$
\sigma_{1}-\sigma_{5}=u_{1}-u_{3}+k_{2}^{1}-k_{2}^{-}>0,
$$

in which $u_{1}>u_{3}$ and $k_{2}^{1}>k_{2}^{-}>0$ have been used. Hence, $S_{1}$ catches up with $S_{5}$ in finite time and the intersection $\left(x_{2}, t_{2}\right)$ is determined by

$$
\begin{gathered}
x_{2}=\sigma_{1} t_{2}, \\
x_{2}-x_{1}=\sigma_{5}\left(t_{2}-t_{1}\right),
\end{gathered}
$$

which yields

$$
\begin{gathered}
\left(x_{2}, t_{2}\right)=\left(\frac{\left(u_{1}+u_{-}+k_{1}^{-}\right)\left(u_{m}-u_{3}+k_{2}^{1}-k_{1}^{1}\right) x_{0}}{\left(u_{1}-u_{2}+u_{m}+2 k_{2}^{1}\right)\left(u_{-}-u_{3}\right)},\right. \\
\left.\frac{\left(u_{m}-u_{3}+k_{2}^{1}-k_{1}^{1}\right) x_{0}}{\left(u_{1}-u_{2}+u_{m}+2 k_{2}^{1}\right)\left(u_{-}-u_{3}\right)}\right) .
\end{gathered}
$$

It can be seen from the $(u, \rho)$ phase plane in Figure 3 that the two states $\left(u_{-}, \rho_{-}\right)$and $\left(u_{3}, \rho_{3}\right)$ can be connected by a shock wave of the first family directly. Thus, after this time $t_{2}$, the two shock waves $S_{1}$ and $S_{5}$ coalesce into a new shock wave which is denoted by $S_{7}$, whose propagation speed is

$$
\sigma_{7}=u_{3}+\frac{u_{-}-\sqrt{u_{-}^{2}+4 \rho_{-}}}{2}=u_{3}-k_{2}^{-} .
$$

It is easy to get $\sigma_{1}>\sigma_{7}>\sigma_{5}$ from $u_{1}>u_{3}$ and $k_{2}^{1}>k_{2}^{-}>0$.

Then, we turn our attention to the interaction between $S_{6}$ and $S_{4}$. The propagation speed of $S_{4}$ is

$$
\sigma_{4}=u_{+}+\frac{u_{2}+\sqrt{u_{2}^{2}+4 \rho_{2}}}{2}=u_{+}-k_{1}^{2} .
$$

Thus, it follows from (33) and (40) that

$$
\sigma_{6}-\sigma_{4}=u_{2}-k_{1}^{3}-u_{+}+k_{1}^{2}>0,
$$

in which $u_{2}>u_{+}$and $k_{1}^{3}<k_{1}^{2}<0$ have been used. As before, $S_{6}$ catches up with $S_{4}$ in finite time and the intersection $\left(x_{3}, t_{3}\right)$ can be calculated by

$$
\begin{gathered}
\left(x_{3}, t_{3}\right)=\left(x_{0}+\frac{\left(u_{+}+u_{2}+k_{2}^{2}\right)\left(u_{3}+k_{2}^{3}+k_{2}^{1}\right) x_{0}}{\left(u_{1}-u_{2}+u_{m}+2 k_{2}^{1}\right)\left(u_{3}-u_{+}\right)},\right. \\
\left.\frac{\left(u_{3}+k_{2}^{3}+k_{2}^{1}\right) x_{0}}{\left(u_{1}-u_{2}+u_{m}+2 k_{2}^{1}\right)\left(u_{3}-u_{+}\right)}\right) .
\end{gathered}
$$



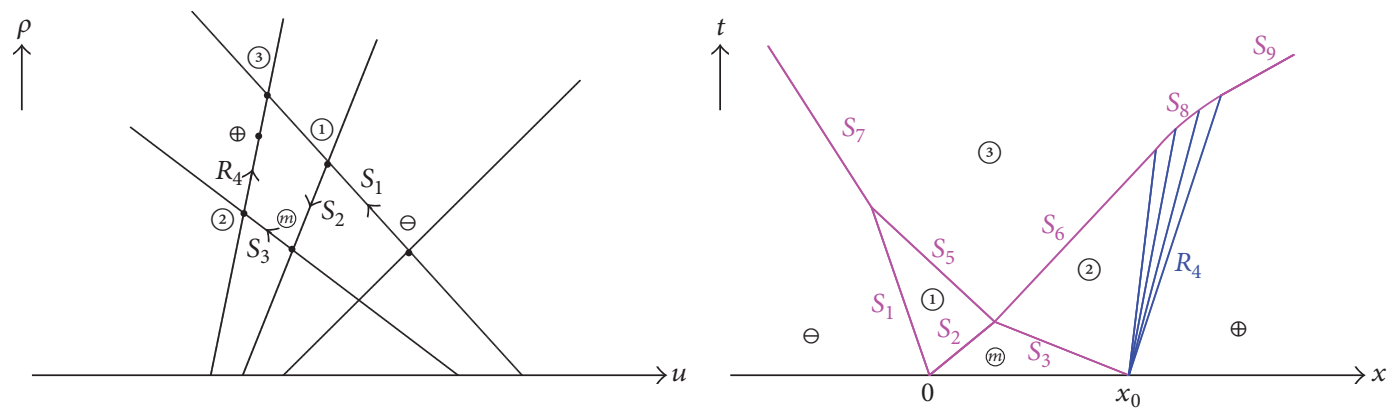

FIgURE 4: The interaction between $S+S$ and $S+R$ is shown when $\left(u_{+}, \rho_{+}\right) \in V_{2}$.

It can also be seen from the $(u, \rho)$ phase plane in Figure 3 that the two states $\left(u_{3}, \rho_{3}\right)$ and $\left(u_{+}, \rho_{+}\right)$can be connected by a shock wave of the second family directly. Thus, after this time $t_{3}$, the two shock waves $S_{6}$ and $S_{4}$ coalesce into a new shock wave which is denoted by $S_{8}$, whose propagation speed is

$$
\sigma_{8}=u_{+}+\frac{u_{3}+\sqrt{u_{3}^{2}+4 \rho_{3}}}{2}=u_{+}-k_{1}^{3} .
$$

In addition, it can also turn out that $\sigma_{6}>\sigma_{8}>\sigma_{4}$ holds from $u_{2}>u_{+}$and $k_{1}^{3}<k_{1}^{2}<0$. In other words, the propagation speed of $S_{8}$ is between those of $S_{4}$ and $S_{6}$. The proof is completed.

Case $2(S+S$ and $S+R)$. In this case, we turn our attention to the situation that the Riemann solution is a shock wave followed by a rarefaction wave starting from the initial point $\left(x_{0}, 0\right)$. We use $S_{3}$ and $R_{4}$ to denote them, respectively (see Figure 4$)$. On this occasion, the state $\left(u_{+}, \rho_{+}\right)$should lie in the region $V_{2} \cup V_{3}$ in Figure 2, which should satisfy the conditions $\rho_{+}>\rho_{m}$ and

$$
\begin{gathered}
u_{m}-\frac{2\left(\rho_{+}-\rho_{m}\right)}{u_{m}+\sqrt{u_{m}^{2}+4 \rho_{m}}}<u_{+} \\
<u_{m}+\frac{2\left(\rho_{+}-\rho_{m}\right)}{\sqrt{u_{m}^{2}+4 \rho_{m}}-u_{m}} .
\end{gathered}
$$

In this situation, the local solution of the perturbed Riemann problem (1) and (2) for the sufficiently small time $t$ may be indicated by the symbols as

$$
\begin{aligned}
& \left(u_{-}, \rho_{-}\right)+S_{1}+\left(u_{1}, \rho_{1}\right)+S_{2}+\left(u_{m}, \rho_{m}\right)+S_{3} \\
& \quad+\left(u_{2}, \rho_{2}\right)+R_{4}+\left(u_{+}, \rho_{+}\right) .
\end{aligned}
$$

Hereafter the intermediate states $\left(u_{1}, \rho_{1}\right),\left(u_{2}, \rho_{2}\right)$, and $\left(u_{3}, \rho_{3}\right)$ have the same presentations as those in Case 1 .

As before, $S_{2}$ collides with $S_{3}$ at the point $\left(x_{1}, t_{1}\right)$ which may be also calculated by the formula (27) in Case 1. After the time $t_{1}$, the new local Riemann problem with the left state $\left(u_{1}, \rho_{1}\right)$ and the right state $\left(u_{2}, \rho_{2}\right)$ can also be solved by two shock waves which are denoted by $S_{5}$ and $S_{6}$, respectively.
Similarly, the two shock waves $S_{1}$ and $S_{5}$ of the first family coalesce into a new shock wave of the first family which is denoted by $S_{7}$.

On the other hand, we consider the situation that the shock wave $S_{6}$ penetrates the rarefaction wave $R_{4}$ which can be summarized below.

Lemma 7. The shock wave $S_{6}$ is able to catch up with the wave back of the rarefaction wave $R_{4}$ in finite time and consequently begins to penetrate $R_{4}$. More precisely, if $u_{+}<u_{3}$, then $S_{6}$ has the ability to cancel the whole $R_{4}$ thoroughly. Otherwise, if $u_{+}>u_{3}$, then $S_{6}$ penetrates $R_{4}$ incompletely and finally has the characteristic line

$$
x=x_{0}+\left(\frac{3 u_{3}+\sqrt{u_{3}^{2}+4 \rho_{3}}}{2}\right) t
$$

in $R_{4}$ as its asymptotic line.

Proof. It is obvious to see that the propagation speed of $S_{6}$ is given by (33) and that of the wave back in the rarefaction wave $R_{4}$ is calculated by

$$
\xi_{4}\left(u_{2}, \rho_{2}\right)=\frac{3 u_{2}+\sqrt{u_{2}^{2}+4 \rho_{2}}}{2}=u_{2}-k_{1}^{2}
$$

In view of $k_{1}^{3}<k_{1}^{2}<0$, we have

$$
\sigma_{6}-\xi_{4}\left(u_{2}, \rho_{2}\right)=k_{1}^{2}-k_{1}^{3}>0,
$$

which implies that $S_{6}$ keeps up with the wave back of $R_{4}$ in finite time and the intersection $\left(x_{3}, t_{3}\right)$ is computed by

$$
\begin{aligned}
& x_{3}-x_{1}=\sigma_{6}\left(t_{3}-t_{1}\right)=\left(u_{2}-k_{1}^{3}\right)\left(t_{3}-t_{1}\right), \\
& x_{3}-x_{0}=\xi_{4}\left(u_{2}, \rho_{2}\right) \cdot t_{3}=\left(u_{2}-k_{1}^{2}\right) t_{3},
\end{aligned}
$$

in which $t_{1}$ is given by (27). Thus, we have

$$
\begin{gathered}
\left(x_{3}, t_{3}\right)=\left(x_{0}+\frac{\left(u_{2}-k_{1}^{2}\right)\left(u_{3}+k_{2}^{3}+k_{2}^{1}\right) x_{0}}{\left(u_{1}-u_{2}+u_{m}+2 k_{2}^{1}\right)\left(u_{3}-u_{2}\right)},\right. \\
\left.\frac{\left(u_{3}+k_{2}^{3}+k_{2}^{1}\right) x_{0}}{\left(u_{1}-u_{2}+u_{m}+2 k_{2}^{1}\right)\left(u_{3}-u_{2}\right)}\right) .
\end{gathered}
$$


Consequently, $S_{6}$ begins to penetrate $R_{4}$ after the time $t_{3}$. It is remarkable that both $S_{6}$ and $R_{4}$ are attributed to the waves of the second family. It can also be seen from the $(u, \rho)$ phase plane in Figure 4 that the state $\left(u_{3}, \rho_{3}\right)$ and the varying state $(u, \rho)$ along with the corresponding characteristic line in $R_{4}$ can also be connected by a shock wave of the second family directly. Thus, during the process of penetration, the shock wave is denoted by $S_{8}$ whose propagation speed is given by

$$
\sigma_{8}=u+\frac{u_{3}+\sqrt{u_{3}^{2}+4 \rho_{3}}}{2},
$$

where $u$ varies from $u_{2}$ to $u_{+}$. Thus, the shock curve of $S_{8}$ in the $(x, t)$ plane is determined by

$$
\begin{aligned}
\frac{d x}{d t} & =u+\frac{u_{3}+\sqrt{u_{3}^{2}+4 \rho_{3}}}{2}, \\
x-x_{0} & =\left(\frac{3 u+\sqrt{u^{2}+4 \rho}}{2}\right) t, \\
x\left(t_{3}\right) & =x_{3} .
\end{aligned}
$$

From the $(u, \rho)$ phase plane in Figure 4 and the notation of $k_{2}$ in this paper, we always have $k_{2}=k_{2}^{3}$ when $u$ varies from $u_{2}$ to $u_{+}$. Thus, we can arrive at

$$
\begin{aligned}
x-x_{0}= & \left(2 u_{3}+k_{2}^{3}\right) t \\
& -2 \sqrt{\frac{\left(u_{3}-u_{2}\right)\left(u_{3}+k_{2}^{1}+k_{2}^{3}\right) x_{0} t}{u_{1}+u_{m}-u_{2}+2 k_{2}^{1}}} .
\end{aligned}
$$

In addition, by differentiating (53) with respect to $t$ twice, we have

$$
\frac{d^{2} x}{d t^{2}}=\frac{1}{2} \sqrt{\frac{\left(u_{3}-u_{2}\right)\left(u_{3}+k_{2}^{1}+k_{2}^{3}\right) x_{0}}{\left(u_{1}+u_{m}-u_{2}+2 k_{2}^{1}\right) t^{3}}}>0,
$$

which means that the shock wave $S_{8}$ speeds up during the process of penetration.

Furthermore, there exist two possible situations according to the region in which the state $\left(u_{+}, \rho_{+}\right)$lies.

(a) If $\left(u_{+}, \rho_{+}\right) \in V_{2}$, then we have $u_{+}<u_{3}$. In this situation, $S_{8}$ is able to cancel $R_{4}$ completely and terminates at the point $\left(x_{4}, t_{4}\right)$ which is given by

$$
\begin{aligned}
x_{4}-x_{0}= & \left(\frac{3 u_{+}+\sqrt{u_{+}^{2}+4 \rho_{+}}}{2}\right) t_{4}, \\
x_{4}-x_{0}= & \left(2 u_{3}+k_{2}^{3}\right) t_{4} \\
& -2 \sqrt{\frac{\left(u_{3}-u_{2}\right)\left(u_{3}+k_{2}^{1}+k_{2}^{3}\right) x_{0} t_{4}}{u_{1}+u_{m}-u_{2}+2 k_{2}^{1}}} ;
\end{aligned}
$$

namely, we have

$$
\begin{aligned}
& \left(x_{4}, t_{4}\right)=\left(x_{0}\right. \\
& +\frac{\left(2 u_{+}+k_{2}^{+}\right)\left(u_{3}-u_{2}\right)\left(u_{3}+k_{2}^{1}+k_{2}^{3}\right) x_{0}}{\left(u_{1}+u_{m}-u_{2}+2 k_{2}^{1}\right)\left(u_{3}-u_{+}\right)^{2}}, \\
& \left.\frac{\left(u_{3}-u_{2}\right)\left(u_{3}+k_{2}^{1}+k_{2}^{3}\right) x_{0}}{\left(u_{1}+u_{m}-u_{2}+2 k_{2}^{1}\right)\left(u_{3}-u_{+}\right)^{2}}\right) .
\end{aligned}
$$

After the penetration, the shock wave is denoted by $S_{9}$ whose propagation speed is

$$
\sigma_{9}=u_{+}+\frac{u_{3}+\sqrt{u_{3}^{2}+4 \rho_{3}}}{2}=u_{+}-k_{1}^{3} .
$$

(b) If $\left(u_{+}, \rho_{+}\right) \in V_{3}$, then we have $u_{+}>u_{3}$. Thus $S_{8}$ cannot cancel the entire rarefaction wave $R_{4}$ thoroughly and ultimately has the characteristic line in $R_{4}$ whose expression is shown in (46) as the asymptotic line.

Case $3(S+S$ and $R+S)$. In this case, we consider that the Riemann solution emanating from the initial point $\left(x_{0}, 0\right)$ is a rarefaction wave followed by a shock wave. This case happens if and only if the conditions $0 \leq \rho_{+}<\rho_{m}$ and

$$
\begin{gathered}
u_{m}+\frac{2\left(\rho_{+}-\rho_{m}\right)}{\sqrt{u_{m}^{2}+4 \rho_{m}}-u_{m}}<u_{+} \\
<u_{m}-\frac{2\left(\rho_{+}-\rho_{m}\right)}{u_{m}+\sqrt{u_{m}^{2}+4 \rho_{m}}}
\end{gathered}
$$

are satisfied. In other words, the state $\left(u_{+}, \rho_{+}\right)$should locate in the region $V_{4} \cup V_{5}$ in Figure 2. We use $R_{3}$ and $S_{4}$ to denote the rarefaction wave and the shock wave, respectively. When $t$ is small enough, the solution of the perturbed Riemann problem (1) and (2) may be displayed in the symbol form

$$
\begin{aligned}
& \left(u_{-}, \rho_{-}\right)+S_{1}+\left(u_{1}, \rho_{1}\right)+S_{2}+\left(u_{m}, \rho_{m}\right)+R_{3} \\
& \quad+\left(u_{2}, \rho_{2}\right)+S_{4}+\left(u_{+}, \rho_{+}\right) .
\end{aligned}
$$

Let us first consider the situation that the shock wave $S_{2}$ penetrates the rarefaction wave $R_{3}$ and use the following lemma to depict it.

Lemma 8. The shock wave $S_{2}$ is able to penetrate the whole rarefaction wave $R_{3}$ thoroughly in finite time and then a transmitted rarefaction wave is generated during the process of penetration.

Proof. It can be seen that the propagation speed of $S_{2}$ is given by (23) and that of the wave back in the rarefaction wave $R_{3}$ is given by

$$
\xi_{3}\left(u_{m}, \rho_{m}\right)=\frac{3 u_{m}-\sqrt{u_{m}^{2}+4 \rho_{m}}}{2}=u_{m}-k_{2}^{m} .
$$


Noticing that $k_{2}^{m}>0>k_{1}^{1}$, we can obtain

$$
\sigma_{2}-\xi_{3}\left(u_{m}, \rho_{m}\right)=k_{2}^{m}-k_{1}^{1}>0,
$$

which means that $S_{2}$ penetrates the wave back of $R_{3}$ at the point which may be computed by

$$
\begin{gathered}
x_{1}=\sigma_{2} t_{1}, \\
x_{1}-x_{0}=\xi_{3}\left(u_{m}, \rho_{m}\right) \cdot t_{1} ;
\end{gathered}
$$

namely,

$$
\left(x_{1}, t_{1}\right)=\left(\frac{\left(u_{m}+u_{1}+k_{2}^{1}\right) x_{0}}{u_{1}+2 k_{2}^{1}}, \frac{x_{0}}{u_{1}+2 k_{2}^{1}}\right) .
$$

After the time $t_{1}$, the shock wave $S_{2}$ begins to penetrate the rarefaction wave $R_{3}$ and is denoted by $S_{6}$ during the process of penetration. This penetration gives rise to a transmitted rarefaction wave which is denoted by $R_{5}$. In order to compare the propagation speeds of rarefaction waves before and after penetration when across $S_{6}$, we have the following description. The propagation speeds of the matched characteristic lines in $R_{3}$ and $R_{5}$ can be calculated, respectively, by

$$
\begin{aligned}
& \xi_{3}\left(u^{-}, \rho^{-}\right)=\frac{3 u^{-}-\sqrt{\left(u^{-}\right)^{2}+4 \rho^{-}}}{2}, \\
& \xi_{5}\left(u^{+}, \rho^{+}\right)=\frac{3 u^{+}-\sqrt{\left(u^{+}\right)^{2}+4 \rho^{+}}}{2},
\end{aligned}
$$

in which the state $\left(u^{-}, \rho^{-}\right)$in $R_{3}$ becomes the matched one $\left(u^{+}, \rho^{+}\right)$in $R_{5}$ when across $S_{2}$. They should obey the relation

$$
\begin{aligned}
\frac{\rho^{+}-\rho^{-}}{u^{+}-u^{-}} & =\frac{\sqrt{\left(u^{-}\right)^{2}+4 \rho^{-}}-u^{-}}{2} \\
& =\frac{\sqrt{\left(u^{+}\right)^{2}+4 \rho^{+}}-u^{+}}{2},
\end{aligned}
$$

in which $u_{m}<u^{-}<u_{2}$ and $u_{1}<u^{+}<u_{3}$. For the match characteristic lines in $R_{3}$ and $R_{5}$, taking into account $u^{-}<u^{+}$, it follows from (64), (65), and (66) that $\xi_{3}\left(u^{-}, \rho^{-}\right)<$ $\xi_{5}\left(u^{+}, \rho^{+}\right)$. That is to say, the rarefaction wave accelerates when it passes through the shock wave $S_{2}$.

On the other hand, during the process of penetration, the curve of the shock wave $S_{6}$ is determined by

$$
\begin{aligned}
\frac{d x}{d t} & =u^{-}+\frac{u^{+}+\sqrt{\left(u^{+}\right)^{2}+4 \rho^{+}}}{2}, \\
x-x_{0} & =\left(\frac{3 u^{-}-\sqrt{\left(u^{-}\right)^{2}+4 \rho^{-}}}{2}\right) t, \\
x\left(t_{1}\right) & =x_{1} .
\end{aligned}
$$

An easy computation leads to

$$
\begin{aligned}
x-x_{0}= & -\left(k_{1}^{m}+2 k_{1}^{1}\right) t \\
& +\left(u_{m}-k_{2}^{1}+k_{1}^{m}+2 k_{1}^{1}\right) \sqrt{\frac{x_{0} t}{u_{1}+2 k_{2}^{1}}} .
\end{aligned}
$$

In addition, the following follows from (68):

$$
\begin{aligned}
\frac{d^{2} x}{d t^{2}} & =-\frac{1}{4}\left(u_{m}-k_{2}^{1}+k_{1}^{m}+2 k_{1}^{1}\right) \sqrt{\frac{x_{0}}{\left(u_{1}+2 k_{2}^{1}\right) t^{3}}} \\
& >0
\end{aligned}
$$

which means that the shock wave $S_{6}$ also speeds up during the process of penetration. It is obvious to see that the shock wave $S_{2}$ is able to penetrate the whole rarefaction wave $R_{3}$ thoroughly in finite time for the reason that the states $\left(u_{3}, \rho_{3}\right)$ and $\left(u_{2}, \rho_{2}\right)$ can be connected directly by a shock wave of the second family which is denoted by $S_{7}$ after penetration. The penetration terminates at the point $\left(x_{2}, t_{2}\right)$ which is given by

$$
\begin{aligned}
x_{2}-x_{0}= & \left(\frac{3 u_{2}-\sqrt{u_{2}^{2}+4 \rho_{2}}}{2}\right) t_{2}, \\
x_{2}-x_{0}= & -\left(k_{1}^{m}+2 k_{1}^{1}\right) t_{2} \\
& +\left(u_{m}-k_{2}^{1}+k_{1}^{m}+2 k_{1}^{1}\right) \sqrt{\frac{x_{0} t_{2}}{u_{1}+2 k_{2}^{1}}} ;
\end{aligned}
$$

namely,

$$
\begin{aligned}
& \left(x_{2}, t_{2}\right)=\left(x_{0}\right. \\
& +\frac{\left(2 u_{2}+k_{1}^{2}\right)\left(u_{m}-k_{2}^{1}+k_{1}^{m}+2 k_{1}^{1}\right)^{2} x_{0}}{4\left(u_{1}+2 k_{2}^{1}\right)\left(u_{2}+k_{1}^{m}+k_{1}^{1}\right)^{2}}, \\
& \left.\quad \frac{\left(u_{m}-k_{2}^{1}+k_{1}^{m}+2 k_{1}^{1}\right)^{2} x_{0}}{4\left(u_{1}+2 k_{2}^{1}\right)\left(u_{2}+k_{1}^{m}+k_{1}^{1}\right)^{2}}\right) .
\end{aligned}
$$

Thus, the conclusion can be drawn and the proof is completed.

The shock wave $S_{7}$ continues to move forward and consequently overtakes the shock wave $S_{4}$ at the point which is given by

$$
\begin{aligned}
& \left(x_{3}, t_{3}\right)=\left(x_{0}\right. \\
& +\frac{\left(u_{+}+u_{2}+k_{2}^{2}\right)\left(x_{2}-x_{0}-\left(u_{2}+u_{3}+k_{2}^{3}\right) t_{2}\right)}{u_{+}-u_{3}}, \\
& \left.\frac{x_{2}-x_{0}-\left(u_{2}+u_{3}+k_{2}^{3}\right) t_{2}}{u_{+}-u_{3}}\right),
\end{aligned}
$$



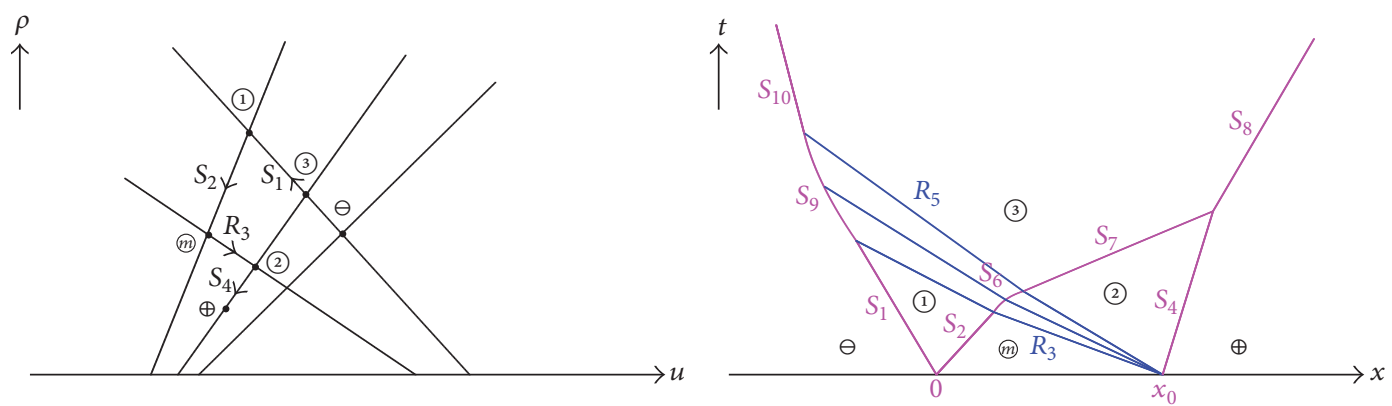

FIgURE 5: The interaction between $S+S$ and $R+S$ is shown when $\left(u_{+}, \rho_{+}\right) \in V_{4}$.

in which $\left(x_{2}, t_{2}\right)$ is given by (71). As before, they also coalesce into a new shock wave denoted by $S_{8}$ due to the fact that the states $\left(u_{3}, \rho_{3}\right)$ and $\left(u_{+}, \rho_{+}\right)$can also be connected directly by a shock wave of the second family. On the other hand, let us consider that the shock wave $S_{1}$ penetrates the rarefaction wave $R_{5}$ which is described in the following lemma.

Lemma 9. If $u_{-}>u_{3}$, then the shock wave $S_{1}$ is able to penetrate the whole rarefaction wave $R_{5}$ thoroughly. Otherwise, if $u_{-}<u_{3}$, then $S_{1}$ penetrates $R_{5}$ incompletely and finally has the characteristic line associated with the state $\left(u_{-}, \rho_{-}\right)$in $R_{5}$ as its asymptotic line.

Proof. The propagation speed of $S_{1}$ and that of the wave back in $R_{5}$ are computed, respectively, by

$$
\begin{gathered}
\sigma_{1}=u_{1}+\frac{u_{-}-\sqrt{u_{-}^{2}+4 \rho_{-}}}{2}=u_{1}-k_{2}^{-}, \\
\xi_{5}\left(u_{1}, \rho_{1}\right)=\frac{3 u_{1}-\sqrt{u_{1}^{2}+4 \rho_{1}}}{2}=u_{1}-k_{2}^{1} .
\end{gathered}
$$

Thus, one can arrive at $\sigma_{1}>\xi_{5}\left(u_{1}, \rho_{1}\right)$ by taking into account $k_{2}^{1}>k_{2}^{-}>0$. Equivalently, the shock wave $S_{1}$ catches up with the wave back of $R_{5}$ in finite time. In fact, the intersection is determined by

$$
\sigma_{1} t_{4}=\xi_{5}\left(u_{1}, \rho_{1}\right) \cdot\left(t_{4}-t_{1}\right)+x_{1}
$$

in which $\left(x_{1}, t_{1}\right)$ is given by (63), which enables us to have

$$
\begin{aligned}
& \left(x_{4}, t_{4}\right)=\left(\frac{\left(u_{1}+u_{-}+k_{1}^{-}\right)\left(u_{m}-u_{1}+k_{2}^{1}-k_{1}^{1}\right) x_{0}}{\left(u_{1}+2 k_{2}^{1}\right)\left(u_{-}-u_{1}\right)},\right. \\
& \left.\quad \frac{\left(u_{m}-u_{1}+k_{2}^{1}-k_{1}^{1}\right) x_{0}}{\left(u_{1}+2 k_{2}^{1}\right)\left(u_{-}-u_{1}\right)}\right) .
\end{aligned}
$$

After the time $t_{4}$, the shock wave begins to penetrate $R_{5}$ with a varying propagation speed and is denoted by $S_{9}$ during the process of penetration. The curve of $S_{9}$ is determined by

$$
\begin{aligned}
\frac{d x}{d t} & =u^{+}+\frac{u_{-}-\sqrt{u_{-}^{2}+4 \rho_{-}}}{2}, \\
x-\bar{x} & =\left(\frac{3 u^{+}-\sqrt{\left(u^{+}\right)^{2}+4 \rho^{+}}}{2}\right)(t-\bar{t}), \\
x\left(t_{4}\right) & =x_{4},
\end{aligned}
$$

in which the point $(\bar{x}, \bar{t})$ is located on the curve of $S_{6}$ and the characteristic line with the state $\left(u^{+}, \rho^{+}\right)$is departing from it. For our knowledge, it is impossible to calculate the explicit form for the curve of $S_{9}$ due to the fact that $R_{5}$ is a noncentered rarefaction wave. Depending on the region in which the state $\left(u_{+}, \rho_{+}\right)$lies, there are two possible situations as follows:

(a) If $\left(u_{+}, \rho_{+}\right) \in V_{4}$, then we have $u_{3}<u_{-}$(see Figure 5). For this subcase, $S_{9}$ is able to cancel the entire $R_{5}$ thoroughly. The shock wave is denoted by $S_{10}$ after penetration whose propagation speed is given by (39).

(b) If $\left(u_{+}, \rho_{+}\right) \in V_{5}$, then we have $u_{3}>u_{-}$(see Figure 6). In this situation, $S_{9}$ cannot penetrate the whole $R_{4}$ completely and at last has the characteristic line associated with the state $\left(u_{-}, \rho_{-}\right)$in $R_{5}$ as its asymptotic line.

Case $4(S+S$ and $R+R)$. In the end, we consider the situation that there are two rarefaction waves originating from the point $\left(x_{0}, 0\right)$. This case arises when the conditions $u_{+}>u_{m}$ and

$$
\begin{gathered}
\max \left(0, \rho_{m}+\frac{\left(-\sqrt{u_{m}^{2}+4 \rho_{m}}-u_{m}\right)\left(u_{+}-u_{m}\right)}{2}\right) \\
<\rho_{+}<\rho_{m}+\frac{\left(\sqrt{u_{m}^{2}+4 \rho_{m}}-u_{m}\right)\left(u_{+}-u_{m}\right)}{2}
\end{gathered}
$$

are satisfied. The two rarefaction waves are denoted by $R_{3}$ and $R_{4}$, respectively. In this case, the state $\left(u_{+}, \rho_{+}\right)$should locate in 

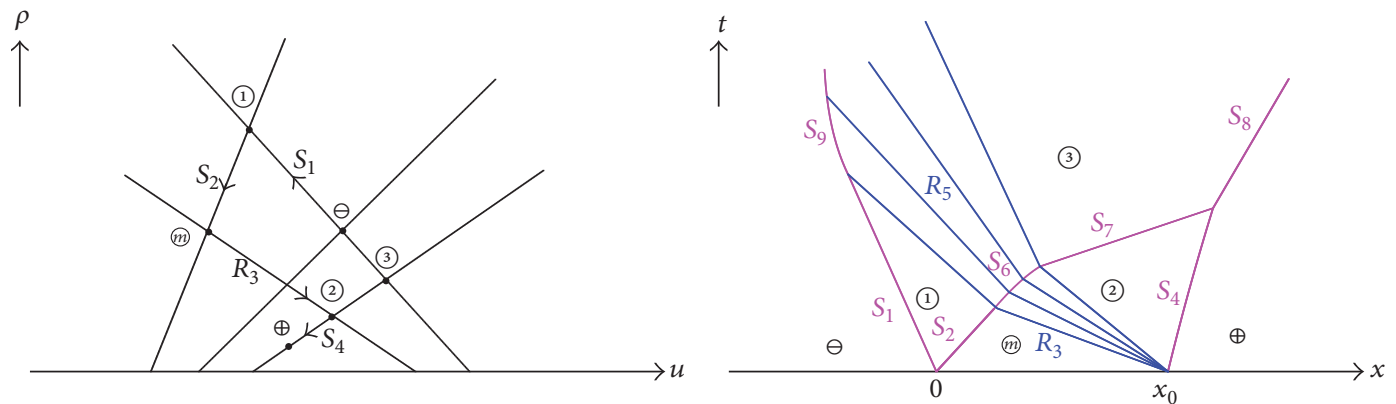

Figure 6: The interaction between $S+S$ and $R+S$ is shown when $\left(u_{+}, \rho_{+}\right) \in V_{5}$.
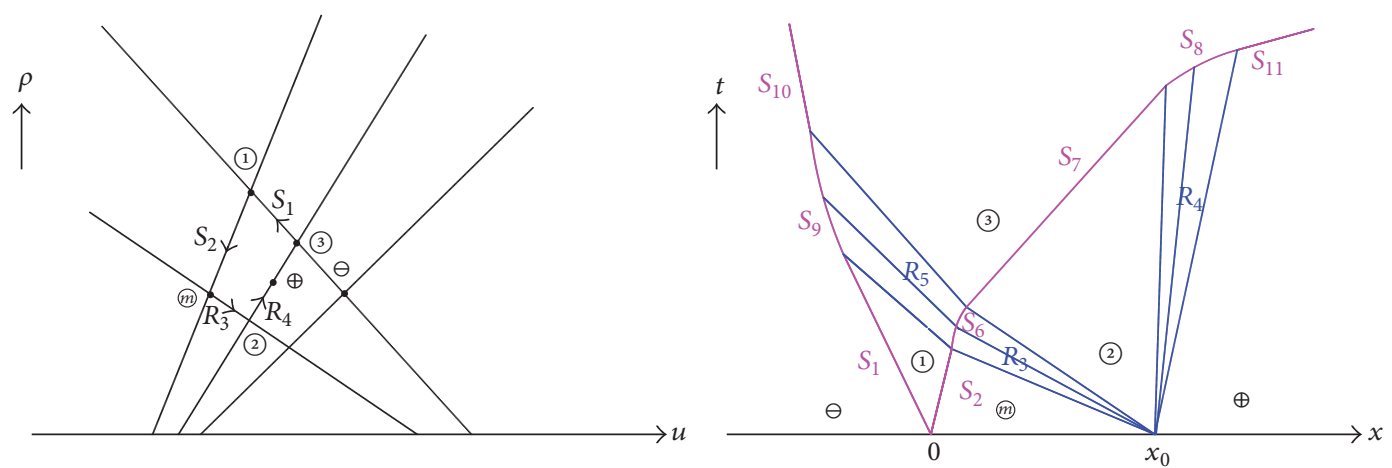

FIgURE 7: The interaction between $S+S$ and $R+R$ is shown when $\left(u_{+}, \rho_{+}\right) \in V_{6}$.

the region $V_{6} \cup V_{7} \cup V_{8} \cup V_{9}$ in Figure 2. The solution of the perturbed Riemann problem (1) and (2) for sufficiently small $t$ may be symbolized as

$$
\begin{aligned}
& \left(u_{-}, \rho_{-}\right)+S_{1}+\left(u_{1}, \rho_{1}\right)+S_{2}+\left(u_{m}, \rho_{m}\right)+R_{3} \\
& \quad+\left(u_{2}, \rho_{2}\right)+R_{4}+\left(u_{+}, \rho_{+}\right) .
\end{aligned}
$$

As in Case 3, the shock wave $S_{2}$ is able to penetrate the entire $R_{3}$ thoroughly in finite time and a transmitted rarefaction wave $R_{5}$ is generated. It can be seen from Lemma 9 that if $u_{-}>u_{3}$, then $S_{1}$ is able to penetrate the whole $R_{5}$ thoroughly. The condition $\left(u_{+}, \rho_{+}\right) \in V_{6} \cup V_{7}$ should be satisfied in this situation. Otherwise, if $u_{-}<u_{3}$, then $S_{1}$ cannot penetrate the whole $R_{5}$ thoroughly. In this situation, $\left(u_{+}, \rho_{+}\right) \in V_{8} \cup V_{9}$ should be satisfied. On the other hand, we turn our attention back to the situation that the shock wave $S_{7}$ penetrates the rarefaction wave $R_{4}$. With the similar process as before, we can see that if $u_{+}<u_{3}$, then $S_{7}$ is able to penetrate the whole $R_{4}$ thoroughly. In this situation, $\left(u_{+}, \rho_{+}\right) \in V_{6} \cup V_{9}$ should be satisfied. Otherwise, if $u_{+}>u_{3}$, then $S_{7}$ cannot penetrate the whole $R_{4}$ completely. In this situation, we need to require $\left(u_{+}, \rho_{+}\right) \in V_{7} \cup V_{8}$.

Thus, this case is just the combination of the results ahead and we omit the details. In fact, we can summarize the main results for the case below.

(a) If $\left(u_{+}, \rho_{+}\right) \in V_{6}$, then we have $u_{+}<u_{3}<u_{-}$(see Figure 7). For this subcase, $S_{1}$ is able to penetrate the whole $R_{5}$ and $S_{7}$ is also able to penetrate the whole $R_{4}$, such that the large-time behavior of solution is $S+S$. (b) If $\left(u_{+}, \rho_{+}\right) \in V_{7}$, then we have $u_{3}<\min \left(u_{-}, u_{+}\right)$. For this subcase, $S_{1}$ is able to penetrate the whole $R_{5}$, but $S_{7}$ cannot penetrate the whole $R_{4}$ completely, such that the large-time behavior of solution is $S+R$.

(c) If $\left(u_{+}, \rho_{+}\right) \in V_{8}$, then we have $u_{-}<u_{3}<u_{+}$(see Figure 8 ). For this subcase, $S_{1}$ cannot penetrate the whole $R_{5}$ and $S_{7}$ also cannot penetrate the whole $R_{4}$, such that the large-time behavior of solution is $R+R$.

(d) If $\left(u_{+}, \rho_{+}\right) \in V_{9}$, then we have $\max \left(u_{-}, u_{+}\right)<u_{3}$. For this subcase, $S_{1}$ cannot penetrate the whole $R_{5}$ completely, but $S_{7}$ is able to penetrate the whole $R_{4}$ thoroughly, such that the large-time behavior of solution is $R+S$.

\section{Conclusion}

So far, the discussion for all kinds of interactions has been accomplished. The global solutions of the perturbed Riemann problem (1) and (2) are constructed completely in explicit forms under Assumption 1. It is clear to see that the largetime asymptotic states of the global solutions to the perturbed Riemann problem (1) and (2) are identical with the corresponding ones to the Riemann problem (1) and (3). In other words, the solutions to Riemann problem (1) and (3) can be obtained if the limits $x_{0} \rightarrow 0$ of the global solutions to the perturbed Riemann problem (1) and (2) are taken. Thus, it turns out that the Riemann solutions are stable with respect to the specific small perturbation (2) of the Riemann initial 

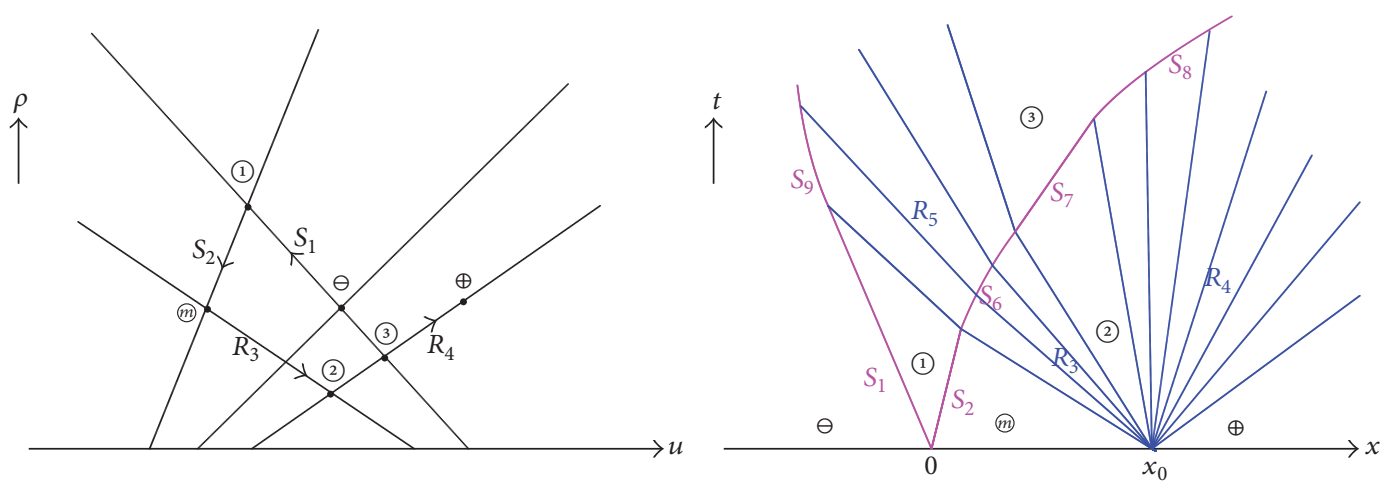

FIgURE 8: The interaction between $S+S$ and $R+R$ is shown when $\left(u_{+}, \rho_{+}\right) \in V_{8}$.

data (3). Based on the above analysis, the proof of Theorem 2 has been finished.

In fact, we can give suitable assumptions such that the Riemann solution originating from the origin $(0,0)$ is $R+S$. By adopting the method developed in this paper, similar results can also be obtained. On the other hand, the reasonable assumptions can also be made such that the Riemann solution originating from the initial point $\left(x_{0}, 0\right)$ is $S+S$ or $S+R$. In this situation, we need to fix the Riemann solution at $\left(x_{0}, 0\right)$ and then change the Riemann solution at $(0,0)$. This is to say, for the given right state $\left(u_{+}, \rho_{+}\right)$, we first fix the intermediate state $\left(u_{m}, \rho_{m}\right)$ and then change the left state $\left(u_{-}, \rho_{-}\right)$. Then, the global solutions to the perturbed Riemann problem (1) and (2) can also be constructed as above. In fact, the above assumptions are made in order to avoid the situation that the forward rarefaction wave collides with the backward one, whose solution cannot be constructed in an explicit form.

\section{Competing Interests}

The authors declare that there is no conflict of interests regarding the publication of this article.

\section{Acknowledgments}

This work is partially supported by National Natural Science Foundation of China (nos. 11441002 and 11271176) and Shandong Provincial Natural Science Foundation (no. ZR2014AM024).

\section{References}

[1] A. Y. Leroux, "Approximation des systems hyperboliques," in Cours et Seminaires INRIA, Problems Hyperboliques, Rocquencourt, 1981.

[2] J. Fritz and B. Tóth, "Derivation of the Leroux system as the hydrodynamic limit of a two-component lattice gas," Communications in Mathematical Physics, vol. 249, no. 1, pp. 1-27, 2004.

[3] V. Popkov and G. M. Schütz, "Why spontaneous symmetry breaking disappears in a bridge system with PDE-friendly boundaries," Journal of Statistical Mechanics: Theory and Experiment, no. 12, Article ID P12004, 2004.

[4] B. Tóth and B. Valkó, "Perturbation of singular equilibria of hyperbolic two-component systems: a universal hydrodynamic limit," Communications in Mathematical Physics, vol. 256, no. 1, pp. 111-157, 2005.

[5] B. Temple, "Systems of conservation laws with invariant submanifolds," Transactions of the American Mathematical Society, vol. 280, no. 2, pp. 781-795, 1983.

[6] E. Canon, "On some hyperbolic systems of temple class," Nonlinear Analysis. Theory, Methods \& Applications. An International Multidisciplinary Journal. Series A: Theory and Methods, vol. 75, no. 11, pp. 4241-4250, 2012.

[7] D. Serre, "Solutions à variations bornées pour certains systèmes hyperboliques de lois de conservation," Journal of Differential Equations, vol. 68, no. 2, pp. 137-168, 1987.

[8] T. Gramchev, "Entropy solutions to conservation laws with singular initial data," Nonlinear Analysis. Theory, Methods \& Applications. An International Multidisciplinary Journal. Series A: Theory and Methods, vol. 24, no. 5, pp. 721-733, 1995.

[9] Y. G. Lu, I. Mantilla, and L. Rendon, "Convergence of approximated solutions to a nonstrictly hyperbolic system," Advanced Nonlinear Studies, vol. 1, pp. 65-79, 2001.

[10] Y.-G. Lu, "Global entropy solutions of Cauchy problem for the Le Roux system," Applied Mathematics Letters. An International Journal of Rapid Publication, vol. 60, pp. 61-66, 2016.

[11] Z. Cheng, "On application of kinetic formulation of the Le Roux system," Proceedings of the Edinburgh Mathematical Society. Series II, vol. 52, no. 1, pp. 263-272, 2009.

[12] G. Chen, E. E. Endres, and H. K. Jenssen, "Pairwise wave interactions in ideal polytropic gases," Archive for Rational Mechanics and Analysis, vol. 204, no. 3, pp. 787-836, 2012.

[13] J. M. Greenberg, "On the elementary interactions for the quasilinear wave equation On the elementary interactions for the quasilinear wave equation $\partial \gamma / \partial t-\partial v / \partial x=0$ and $\partial v / \partial t-$ $\partial \sigma(\gamma) / \partial x=0$," Archive for Rational Mechanics and Analysis, vol. 43, pp. 325-349, 1971.

[14] M. Sun, "Interactions of elementary waves for the Aw-Rascle model," SIAM Journal on Applied Mathematics, vol. 69, no. 6, pp. 1542-1558, 2009.

[15] T. Chang and L. Hsiao, "The Riemann problem and interaction of waves in gas dynamics," in Pitman Monographs and Surveys in Pure and Applied Mathematics, vol. 41 of Longman Scientific and Technical, 1989.

[16] J. Glimm, “The interaction of nonlinear hyperbolic waves," Communications on Pure and Applied Mathematics, vol. 41, no. 5, pp. 569-590, 1988. 
[17] G. Lai, "Interaction of jump-fan composite waves in a twodimensional jet for van der Waals gases," Journal of Mathematical Physics, vol. 56, no. 6, Article ID 061504, 2015.

[18] A. Mentrelli, T. Ruggeri, M. Sugiyama, and N. Zhao, "Interaction between a shock and an acceleration wave in a perfect gas for increasing shock strength," Wave Motion, vol. 45, no. 4, pp. 498-517, 2008.

[19] T. Raja Sekhar and V. D. Sharma, "Riemann problem and elementary wave interactions in isentropic magnetogasdynamics," Nonlinear Analysis. Real World Applications. An International Multidisciplinary Journal, vol. 11, no. 2, pp. 619-636, 2010.

[20] T. T. Li, Global Classical Solutions for Quasilinear Hyperbolic Systems, vol. 32, John Wiley \& Sons, New York, NY, USA, 1994.

[21] C. Shen and M. Sun, "Interactions of delta shock waves for the transport equations with split delta functions," Journal of Mathematical Analysis and Applications, vol. 351, no. 2, pp. 747755, 2009.

[22] L. Guo, Y. Zhang, and G. Yin, "Interactions of delta shock waves for the Chaplygin gas equations with split delta functions," Journal of Mathematical Analysis and Applications, vol. 410, no. 1, pp. 190-201, 2014.

[23] A. Qu and Z. Wang, "Stability of the Riemann solutions for a Chaplygin gas," Journal of Mathematical Analysis and Applications, vol. 409, no. 1, pp. 347-361, 2014.

[24] L. Guo, L. Pan, and G. Yin, "The perturbed Riemann problem and delta contact discontinuity in chromatography equations," Nonlinear Analysis. Theory, Methods \& Applications. An International Multidisciplinary Journal. Series A: Theory and Methods, vol. 106, pp. 110-123, 2014.

[25] C. Shen, "Wave interactions and stability of the Riemann solutions for the chromatography equations," Journal of Mathematical Analysis and Applications, vol. 365, no. 2, pp. 609-618, 2010.

[26] C. Shen, "The asymptotic behaviors of solutions to the perturbed Riemann problem near the singular curve for the chromatography system," Journal of Nonlinear Mathematical Physics, vol. 22, no. 1, pp. 76-101, 2015.

[27] M. Sun, "Interactions of delta shock waves for the chromatography equations," Applied Mathematics Letters, vol. 26, no. 6, pp. 631-637, 2013.

[28] A. Bressan, Hyperbolic Systems of Conservation Laws: The OneDimensional Cauchy Problem, vol. 20 of Oxford Lecture Series in Mathematics and Its Applications, Oxford University Press, Oxford, UK, 2000.

[29] C. M. Dafermos, "Hyperbolic conversation laws in continuum physics," in Grundlehren der Mathematischen Wissenchaften, Springer, Berlin, Germany, 2000.

[30] D. Serre, Systems of Conversation Laws 1/2, Cambridge University Press, Cambridge, UK, 1999/2000.

[31] J. Smoller, Shock Waves and Reaction-Diffusion Equations, Springer, New York, NY, USA, 1994. 


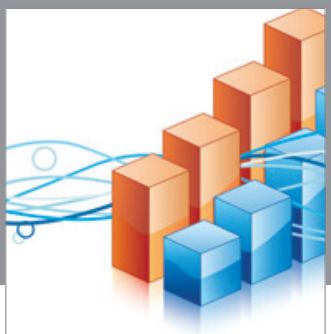

Advances in

Operations Research

vatem alat4

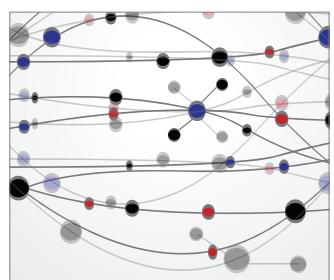

\section{The Scientific} World Journal
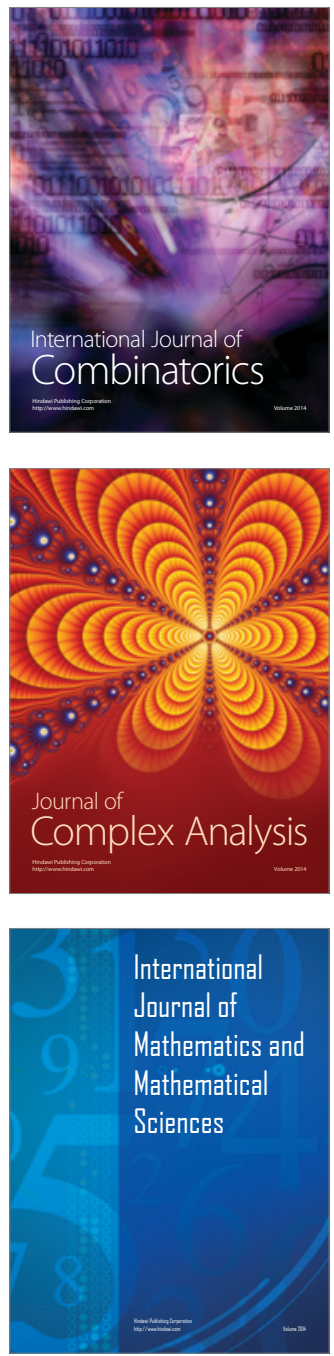
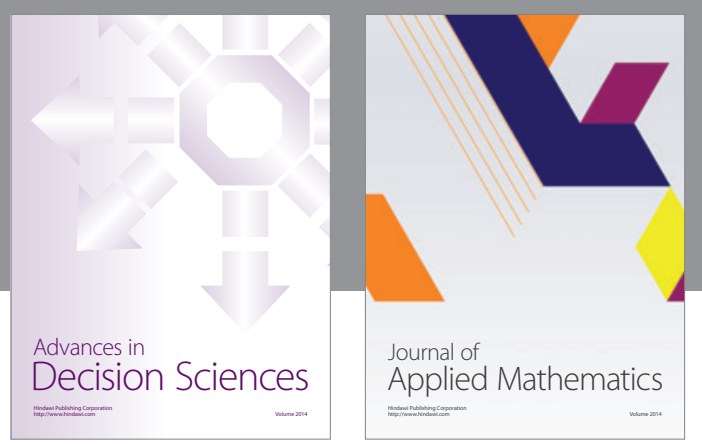

Algebra

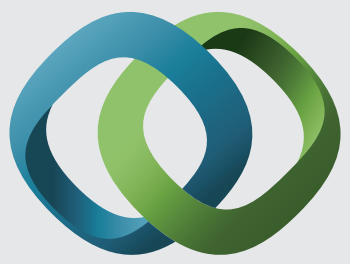

\section{Hindawi}

Submit your manuscripts at

http://www.hindawi.com
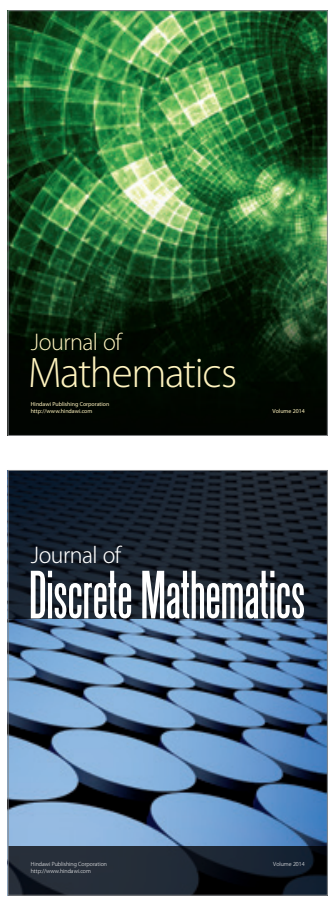

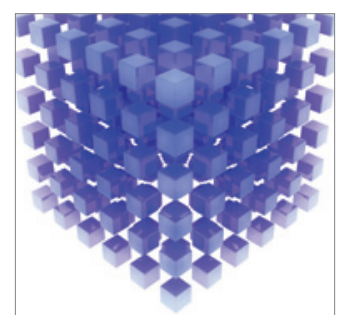

Mathematical Problems in Engineering
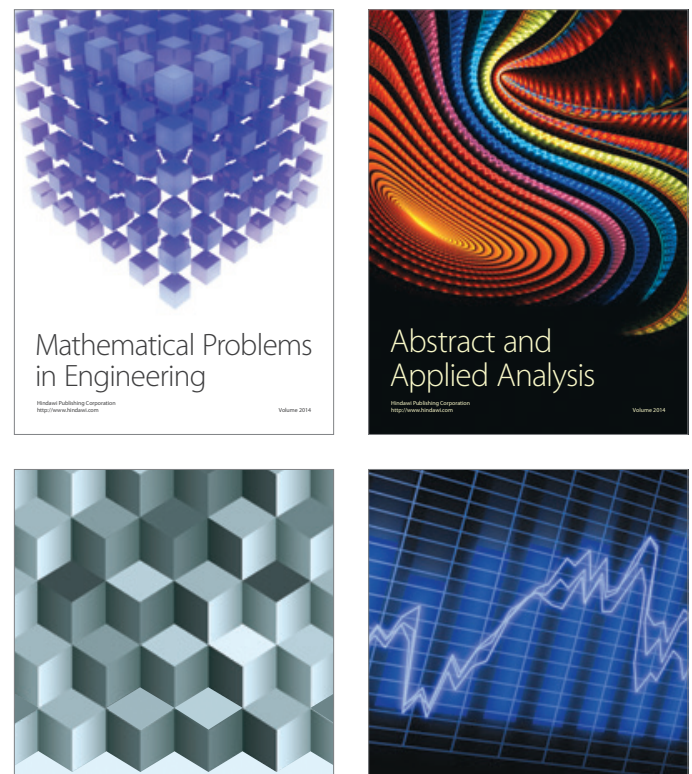

Journal of

Function Spaces

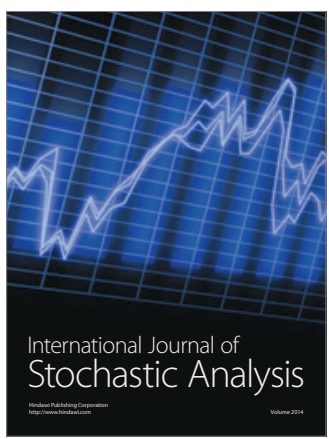

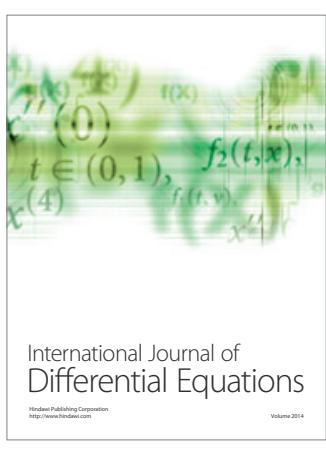
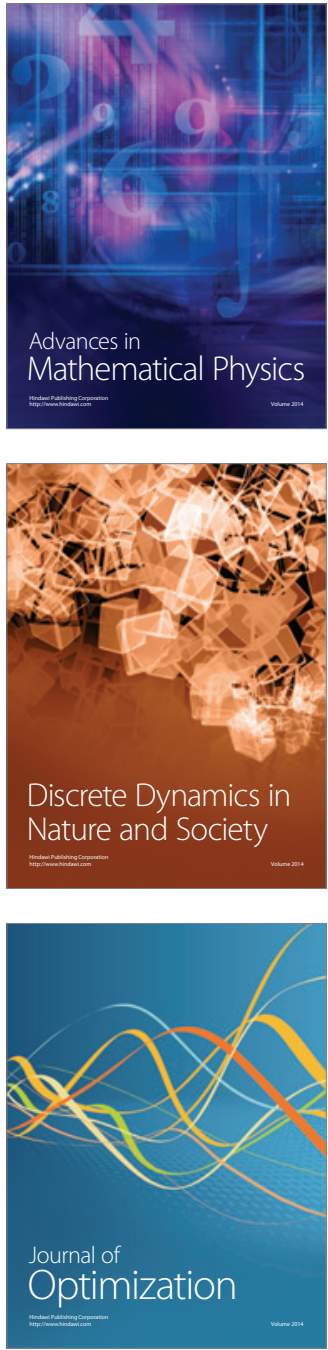